\title{
Comportamiento de la biomasa y de las tasas de crecimiento de dos variedades de lulo (Solanum quitoense Lam.) en Pachavita, Boyacá
}

\section{Biomass and growth rates' behavior of two varieties of lulo (Solanum quitoense Lam.) in Pachavita-Boyacá}

Fecha recepción: 18 de junio de 2015

Fecha aceptación: 22 de diciembre de 2015
Pedro José Almanza-Merchán ${ }^{1}$

Yeily Paola Tovar-León ${ }^{2}$ Julián David Velandia-Díaz ${ }^{3}$

\section{Resumen}

Se estudió la dinámica del crecimiento y desarrollo del fruto de dos variedades de lulo (chonto grueso y criollo) bajo las condiciones del municipio de Pachavita (Boyacá). Se evaluaron la biomasa seca y las tasas de Crecimiento Absoluto (TAC) y de Crecimiento Relativa (TRC). Los frutos de las dos variedades presentaron crecimiento tipo sigmoide simple, con tres fases que se describieron como: FI (división celular), FII (elongación celular) y FIII (maduración). La variedad Chonto grueso mostró un incremento exponencial en la TAC, que abarcó la FI y parte de la FII, hasta los 78 días después de antesis (dda), cuando alcanzó su mayor valor, de 0,117 g/día, y una rápida ganancia de masa seca hasta este punto; luego disminuyó hasta la cosecha. La variedad Criollo mostró un aumento lento en la FI, que fue progresivo hasta llegar a su máximo valor a los $128 \mathrm{dda}$, acumulando 0,107 g/día, lo que implicó un aumento de tamaño más lento en el tiempo; demostrando mayor poder vertedero inicial en la variedad Chonto grueso, respecto a la variedad Criollo, en términos de materia seca. La TRC para la variedad Chonto grueso mostró una lenta disminución en la FI, reduciéndose drásticamente en la Fll y lentamente en la FIII; en tanto que la variedad Criollo mostró un descenso gradual y constante hasta los 140 dda. Se estableció, para la variedad Chonto grueso en la FIII, y en general para la variedad Criollo, una baja tasa de variación del tamaño por unidad de tamaño inicial.

Palabras clave: biomasa; maduración; lulo; solanáceas.

$1 \quad$ Ph.D. Universidad Pedagógica y Tecnológica de Colombia (Tunja, Colombia).pedro.almanza@uptc.edu.co.

2 Profesional Independiente.

3 Profesional Independiente. 


\begin{abstract}
The two fruit varieties growth and development dynamics of lulo (Chonto grueso and Criollo) was studied under conditions of Pachavita municipality, of Boyacá department. Dry biomass, the Absolute Growth Rates (AGR) and Relative Growth (RGR) were evaluated. The two fruits varieties have a simple sigmoid growth, with three phases which were described as: FI (cell division), FII (cell elongation) and FIII (maturation). The Chonto grueso variety showed an exponential increase in the AGR covering the FI and part of the FII, up to 78 days after anthesis (DAA) when it reached its highest value of $0.117 \mathrm{~g} /$ day, and a rapid dry mass gain, up to this point, then decreased until harvest. The Criollo variety showed a slow increase in the $\mathrm{FI}$, which was progressive until reaching its peak at $128 \mathrm{DAA}$, accumulating $0.107 \mathrm{~g} /$ day, which meant a slower increase in size over time, demonstrating greater initial landfill power in Chonto grueso variety, regarding the Criollo variety in terms of dry matter. The RGR for Chonto grueso variety showed a slow decline in FI, drastically reduced in FII and slowly in the FIII; while the Criollo variety showed a gradual and steady decline to 140 DAA. It was established for the Chonto grueso variety in FIII and in general for the Criollo variety, a low rate of change in size per unit initial size.
\end{abstract}

Keywords: biomass; maturation; lulo; solanaceae. 


\section{Introducción}

El lulo (Solanum quitoense Lam.) es un frutal originario de los Andes, que ha sido cultivado y consumido principalmente en Colombia, Ecuador, Panamá y Costa Rica (1, 2); en Colombia se cultiva en los departamentos de Huila, Valle del Cauca, Antioquia, Tolima, Santander, Caldas, Nariño y Boyacá, pero también se encuentran cultivos en otros ocho departamentos (3). Debido a su valor nutritivo, sabor, color y posibilidades de uso en la agroindustria, el lulo es una de las frutas más apetecidas en los mercados nacionales e internacionales, con amplias perspectivas para la exportación a los mercados de Estados Unidos, Japón y la Comunidad Europea $(4,5)$.

Los modelos de crecimiento son herramientas valiosas para entender el comportamiento de sistemas complejos (6). Con el fin de comprender la dinámica del crecimiento y desarrollo y del tamaño de los frutos, se han propuesto modelos ecofisiológicos que describen los procesos del crecimiento de frutos $(7,8,9)$; ante esto, se ha propuesto un modelo simple de actividad de proliferación celular de crecimiento de frutos de tomate bajo condiciones ambientales constantes (10); dicho modelo asume una fase exponencial de proliferación celular que disminuye a medida que avanza la división.

Además, se han venido aplicando análisis de regresión para el ajuste de modelos de crecimiento de frutos, arrojando buenos resultados, con modelos de regresión no lineal y modelos polinómicos de tercer grado. Diferentes autores $(11,12,13)$ propusieron que, si el modelo lineal no es el adecuado, se debe considerar el ajuste de algún modelo no lineal. A su vez, el modelo logístico puede expresar adecuadamente el crecimiento o desarrollo en función del tiempo, que se caracteriza por presentar una curva de forma sigmoidal con un punto de inflexión y dos asíntotas, una superior y otra inferior (14).

Las gráficas del crecimiento han sido útiles para describir los cambios que se presentan en el crecimiento de tejidos vegetales, pues las curvas de crecimiento han reflejado el comportamiento de la planta en un ecosistema en particular respecto al tiempo (15); por lo tanto, su elaboración es importante para realizar una adecuada aplicación de las medidas culturales de manejo. Una curva sigmoide simple representa el tamaño acumulado del fruto en función del tiempo desde la antesis hasta que alcanza su madurez, y comprende tres fases: una primera fase logarítmica, en la que el tamaño aumenta de forma exponencial a través del tiempo, y se caracteriza por una velocidad lenta de crecimiento al principio; una segunda fase lineal, donde el aumento de tamaño es continuo a una velocidad constante, y una tercera fase de senescencia, que se caracteriza por una velocidad decreciente de crecimiento a medida que el fruto alcanza su madurez y empieza a envejecer (16).

El crecimiento es definido como un aumento irreversible en el volumen o la masa de un organismo vivo, acompañado de procesos como la morfogénesis y la diferenciación celular. A su vez, el desarrollo del fruto es un proceso que involucra fenómenos de crecimiento y diferenciación celular, e implica el desarrollo coordinado de un gran número de tejidos (17). Al respecto, (18) afirma que el tamaño final de los frutos carnosos está determinado por tres procesos importantes: la multiplicación celular, que es responsable de la formación del ovario antes de la antesis; la división celular, que tiene lugar después de la antesis y la polinización, y la extensión celular.

El análisis del crecimiento de una planta individual se realiza, generalmente, en estadios tempranos de desarrollo, en los cuales se calculan índices como Tasa Relativa de crecimiento (TRC), Tasa Absoluta de Crecimiento (TAC), Tasa de Asimilación Neta (TAN), Área Foliar Específica (AFE) y Tasa Foliar (19). La TAC indica el cambio de tamaño por unidad de tiempo y ofrece una visión acertada del poder vertedero de los frutos, en términos de acumulación de materia seca; mientras que la TRC expresa la tasa de variación del tamaño por unidad de tamaño inicial $(20,21,22)$. El análisis de crecimiento de plantas puede basarse en la evolución cronológica de medidas como longitud de tallos, número de hojas, número de ramas, etc. (23); mientras las mediciones de peso seco, 
longitud, etc., tienen que ver con el desarrollo absoluto de la planta; estas variables explican su eficiencia para acumular materia seca en los diferentes órganos, como resultado de los procesos metabólicos (24).

Los estudios de crecimiento de frutos (principalmente relacionados con el aumento irreversible en tamaño y peso seco) y de desarrollo (cambios graduales en tamaño, estructura y función) son importantes para evaluar las etapas óptimas de maduración $(25,26,27)$, determinando el comportamiento del crecimiento a través del tiempo, la estimación del tamaño del fruto (28) y del peso (29) en la cosecha, con lo que se proponen estrategias de manejo agrícola $(30,31)$, para finalmente establecer etapas fenológicas y analizar la formación del fruto y el desarrollo estructural (32).

Existen dos metodologías para efectuar el análisis de crecimiento: el análisis tradicional o clásico, que involucra la toma de datos en función del tiempo en un gran número de muestras (19), con los cuales se generan funciones paramétricas flexibles que describen y explican el crecimiento y desarrollo de las plantas, así como la elaboración de curvas de crecimiento; y el análisis funcional o dinámico, que comprende medidas a intervalos de tiempos más frecuentes y en un pequeño número de plantas (33).

El objetivo del presente estudio fue determinar el comportamiento de la biomasa seca y las tasas absoluta y relativa de crecimiento de dos variedades de lulo (Solanum quitoense Lam.), bajo las condiciones agroclimáticas del municipio de Pachavita (Boyacá).

\section{Materiales y métodos}

La colecta de los frutos de lulo se realizó en el municipio de Pachavita, vereda Sacaneca, a 2.148 msnm, con una temperatura media de $17^{\circ} \mathrm{C}$; para ello se seleccionaron 15 plantas de las variedades Chonto grueso y Criollo. Las plantas tenían una edad de 3 años, con distancia de plantación de $2 \times 1,5 \mathrm{~m}$. El manejo agronómico del cultivo correspondió a los empleados por el productor. De cada planta, se marcaron frutos de 30 días después de la antesis (dda), para conformar un total de 120 unidades de muestreo. La colecta se realizó con una frecuencia de 10 días, para un total de 12 muestreos, tomando 4 frutos al azar de cada material, los cuales se almacenaron en bolsas de papel y se trasladaron, bajo condiciones de baja temperatura, al Laboratorio de Fisiología Vegetal de la Universidad Pedagógica y Tecnológica de Colombia, a la mayor brevedad, con el fin de reducir el deterioro de ellos. La colecta se realizó hasta el momento en que las bayas adquirieran los atributos requeridos para su consumo, definiéndolo como 'punto óptimo de cosecha'. La identificación de las variedades se sustentó en la investigación realizada previamente, para caracterizar la diversidad genética en cuatro variedades de lulo en el municipio de Pachavita (34).

La biomasa seca se determinó sometiendo los frutos a secado en una estufa universal Memmert 500 alemán, durante 48 horas, a $85^{\circ} \mathrm{C}, \mathrm{y}$ posteriormente se pesó mediante una balanza de precisión de 0,01 g (Ohaus). Las Tasas Absolutas de Crecimiento (TAC) y Relativa de Crecimiento (TRC) se establecieron a través de las ecuaciones establecidas para determinar el crecimiento básico de plantas (Tabla I) (20).

Tabla I. Ecuaciones utilizadas para determinar TAC y TRC durante el crecimiento y desarrollo de frutos de lulo variedad Chonto grueso y Criollo bajo condiciones agroecológicas del municipio de Pachavita Boyacá.

\begin{tabular}{llll}
\hline Índice & Descripción & Fórmula & Unidades \\
\hline TAC & Tasa Absoluta de Crecimiento & $(\mathrm{dW} / \mathrm{dt})$ & $\mathrm{g} \mathrm{d}^{-1}$ \\
TRC & Tasa Relativa de Crecimiento & $(1 / \mathrm{W})(\mathrm{dW} / \mathrm{dt})$ & $\mathrm{g} \mathrm{g}^{-1} \mathrm{~d}^{-1}$ \\
\hline
\end{tabular}

W: masa seca total (g); dW/dt: Variación de masa seca en función del tiempo. 


\section{Análisis estadístico}

Con los datos obtenidos se determinó el promedio y el error estándar y se realizó un análisis de varianza (ANOVA), para establecer las diferencias estadísticas a una significancia $\leq 0,05$, mediante la utilización del software SPSS $8.0^{\circledR}$. Se graficó el comportamiento de cada una de las variables respecto al tiempo y se determinaron los modelos estadísticos más adecuados. Las tasas de crecimiento y los modelos logísticos fueron determinados mediante análisis funcional, con la utilización del software SAS v. 9.2.

\section{Resultados y discusión}

\section{Biomasa Seca Total}

A partir de las observaciones realizadas y al analizar los datos obtenidos se encontró que el comportamiento de la curva de la masa seca de frutos de lulo Chonto grueso y Criollo fue de tipo sigmoide simple, la cual se ajustó a un modelo de crecimiento tipo logístico (Tabla II).

Tabla II. Ecuaciones de ajuste al modelo logístico para la masa seca, durante el crecimiento y desarrollo de frutos de lulo variedad Chonto grueso y Criollo bajo condiciones agroecológicas del municipio de

Pachavita, Boyacá.

\begin{tabular}{|c|c|c|}
\hline Material & Modelo logístico & $\mathbf{R}^{\mathbf{2}}$ \\
\hline Variedad Chonto grueso & $\mathrm{y}=7,7561 / 1+\mathrm{e}^{-0,0604^{*}(\mathrm{ddd}-78,4801)}$ & 0,99 \\
\hline Variedad Criollo & $\mathrm{y}=14,3088 / 1+\mathrm{e}^{-0,03^{*}(\mathrm{ddd}-128,1)}$ & 0,99 \\
\hline
\end{tabular}

En la Figura 1 se aprecian las tres fases de crecimiento que han sido descritas por (16) y (35).

también ha sido reportada en frutos de tomate cv Esta curva es propia de frutos carnosos (16), y cv 'Quindío' $(22,32)$.

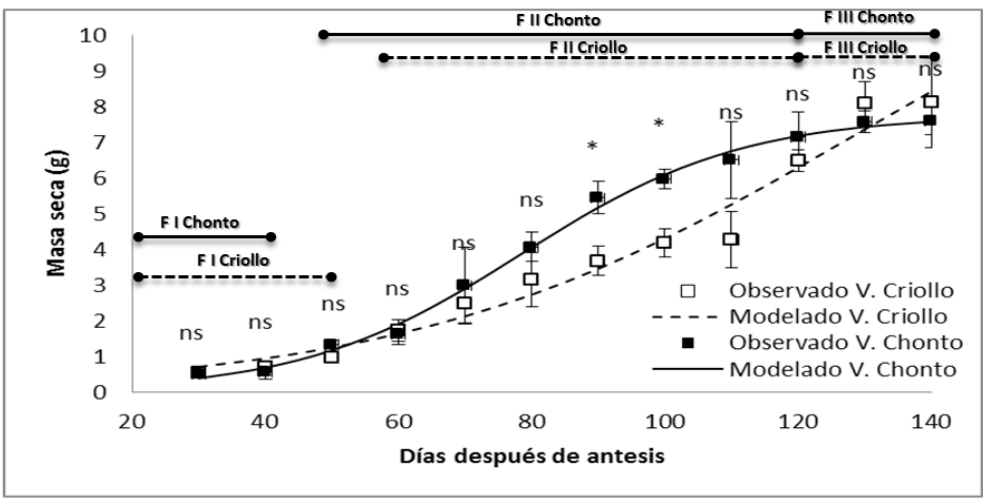

Figura 1. Comportamiento de la masa seca durante el crecimiento y desarrollo de frutos de lulo variedad Chonto grueso y Criollo bajo condiciones agroecológicas del municipio de Pachavita, Boyacá. **Diferencias significativas al 1\%, *Diferencias significativas al 5\%; ns: No hay diferencias estadísticas, de acuerdo con el ANOVA. Las barras verticales indican el error estándar $(n=4)$. F I. División celular; F II. Elongación celular; F III. Maduración.

FASE I (FI). Corresponde a un período de crecimiento visiblemente lento, en el que predominan procesos de rápida división celular (36); se caracterizó por una baja acumulación de masa y por la limitada cantidad de agua en el fruto.
Para la variedad Chonto grueso, esta fase se inició desde la antesis hasta los 40 dda, acumulando $0,5675 \pm 0,19$ g de masa seca; mientras que para la variedad Criollo tuvo una duración de $50 \mathrm{dda}$, con acumulación de 0,985 $\pm 0,03$ g de masa seca, 
valores atribuidos al predominio de procesos de división celular (35).

FASE II (FII). Corresponde al Ilenado del fruto; se presentó un incremento exponencial en el crecimiento con un aumento rápido en la acumulación de biomasa, con predominio de la elongación celular y, posiblemente, una reducción de la intensidad respiratoria (37); tuvo una duración de 80 días. Esta fase finalizó a los 120 dda, mostrando en este punto un peso en biomasa seca de 7,14 $\pm 0,6 \mathrm{~g}$ para la variedad Chonto grueso, y 6,4 $\pm 0,3 \mathrm{~g}$ para la variedad Criollo. De acuerdo con (38), el aumento de la masa seca del fruto de lulo depende de la formación de proteínas, azúcares y otras sustancias acumuladas durante su crecimiento y desarrollo.

FASE III (FIII). Es considerada la fase de maduración; se caracterizó por ser muy corta para las dos variedades (20 días), presentándose a los 130 dda un aumento de la masa seca de $7,58 \pm 0,3$ g para la variedad Chonto grueso y de $8,07 \pm 0,6$ g para la variedad Criollo. Los valores de masa seca se estabilizaron a los 140 dda, para un peso final de $7,6 \pm 0,4$ g y $8,1 \pm 1,2 \mathrm{~g}$, respectivamente. Cabe destacar que, aunque la masa seca siguió aumentando hasta los 130 dda, los cambios que acompañan a la maduración en la fase FIII iniciaron desde los $120 \mathrm{dda}$, sugiriendo que aún en la madurez hay incremento en la biomasa seca de las variedades evaluadas, hasta llegar a un punto de equilibrio. Además, para la variedad Criollo no se apreció la fase asintótica, lo cual, posiblemente, se debe a una continua acumulación de fotoasimilados en la última etapa de crecimiento (18), comportamiento que también fue reportado para champa (Campomanesia lineatifolia Ruiz\&Pav.) (39).

De igual manera, se observaron diferencias significativas al $5 \%$ a los 90 y 100 dda, debido a la mayor ganancia de masa seca por la variedad Chonto grueso, que mostró un incremento exponencial, mientras que la variedad Criollo mantuvo un incremento casi lineal y uniforme.

\section{Tasa Absoluta de Crecimiento}

Las tasas de crecimiento muestran el comportamiento típico para un fruto de lulo como el encontrado en manzana (40) y pera (41), pues la variedad Chonto grueso mostró un incremento exponencial en la TAC que abarcó la FI y parte de la FII, hasta los $78 \mathrm{dda}$, cuando presentó su mayor valor, de $0,117 \mathrm{~g} \mathrm{~d}^{-1}$, señalando una rápida ganancia de masa seca hasta este punto (Figura 2), lo que coincide con lo encontrado para frutos de champa (Campomanesia lineatifolia R \& P) (42); posteriormente, se observó una disminución hasta la cosecha.

Por su parte, la variedad Criollo mostró un aumento lento en la $\mathrm{FI}$ que fue progresivo hasta llegar a su máximo valor a los 128 dda, cuando acumuló $0,107 \mathrm{~g} \mathrm{~d}^{-1}$ (FII), señalando con ello un cambio de tamaño más lento en el tiempo (Figura 2). Esto demuestra el mayor poder vertedero de la variedad Chonto grueso en términos de materia seca (41). 


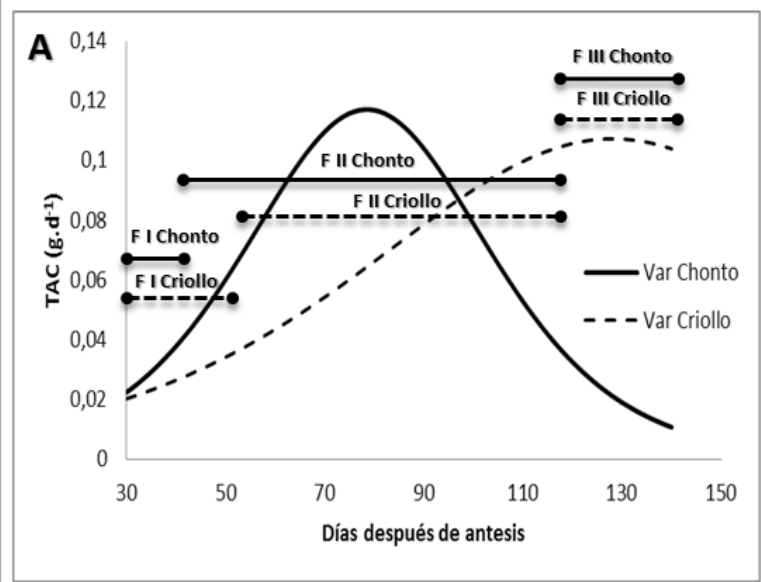

Figura 2. Comportamiento de la Tasa Absoluta de Crecimiento (TAC) durante el crecimiento y desarrollo de frutos de lulo variedad Chonto grueso y Criollo, bajo las condiciones agroecológicas del municipio de Pachavita, Boyacá.

\section{Tasa Relativa de Crecimiento}

En la variedad Chonto grueso, la Tasa Relativa de Crecimiento (TRC) inició con un valor de 0,058 $\mathrm{g} \mathrm{g}^{-1} \mathrm{~d}^{-1}$, mostrando una disminución lenta en la fase I de crecimiento, seguida por una disminución drástica en la fase II, hasta la cosecha, en la fase de crecimiento III (Figura 3), similar a lo encontrado en frutos de champa (Campomanesia lineatifolia $R$ \& P) por (42) y (41) en pera (2015), quienes reportan que la disminución es constante durante todo el crecimiento del fruto. En tanto, la variedad Criollo inició con $0,030 \mathrm{~g} \mathrm{~g}^{-1} \mathrm{~d}^{-1} \mathrm{y}$ empezó un descenso gradual y constante hasta los $140 \mathrm{dda}$, correspondiente a la FIII; datos similares fueron reportados por (40). Se destaca que para la variedad Chonto grueso, en la FIII, y en general para la variedad Criollo, se presenta una baja tasa de variación del tamaño inicial por unidad (20), lo que para la variedad Chonto grueso concuerda con el bajo crecimiento que se da durante los procesos de maduración.

Además, la variedad Chonto grueso superó a la variedad Criollo en los valores de TRC hasta los $86 \mathrm{dda}$, indicando que es más eficiente en la producción de masa seca hasta ese período; sin embargo, a partir de este punto la drástica reducción de la TRC en la variedad Chonto grueso y la disminución gradual en la variedad Criollo invirtieron la relación, lo cual se correlaciona con el incremento continuo y constante de masa seca en la variedad Criollo, que explica una continua acumulación de asimilados en la última etapa de crecimiento (18).

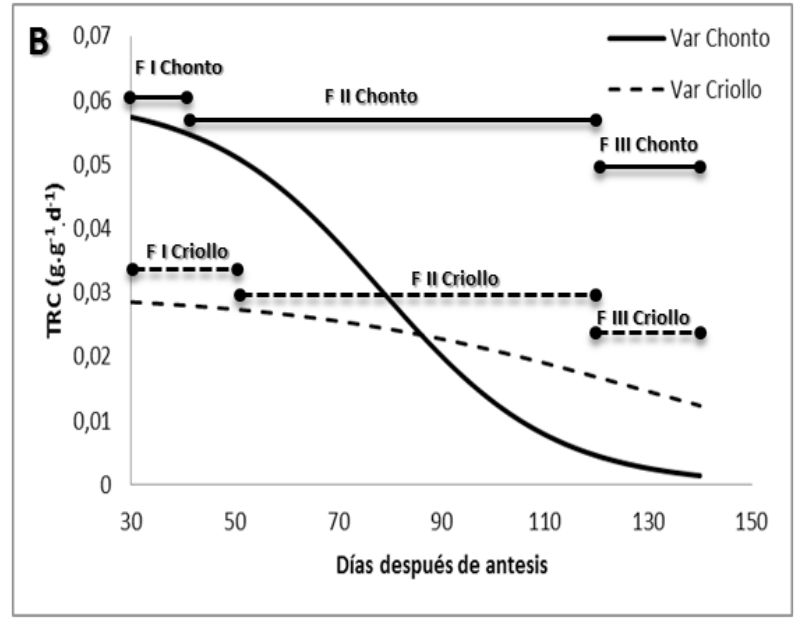

Figura 3. Comportamiento de la Tasa Relativa de Crecimiento (TRC) durante el crecimiento y desarrollo de frutos de lulo variedad Chonto grueso y Criollo, bajo las condiciones agroecológicas del municipio de Pachavita, Boyacá.

\section{Conclusiones}

La TAC para la variedad Chonto grueso mostró un comportamiento de campana típico de la mayoría de frutales en cuanto a su crecimiento, señalando un incremento exponencial hasta los $78 \mathrm{dda}$, con lo que indica una rápida ganancia de masa seca hasta este punto y disminuyendo posteriormente hasta la cosecha. Por su parte, la variedad Criollo mostró un aumento lento y progresivo hasta los $128 \mathrm{dda}$, señalando con ello un cambio de tamaño más lento en el tiempo. Esto demuestra el mayor poder vertedero de la variedad Chonto grueso en términos de materia seca.

La variedad Chonto grueso superó a la variedad Criollo en los valores de TRC, indicando que es más eficiente en la producción de masa seca hasta los 86 dda, pues a partir de este punto se dio el incremento continuo y constante de masa seca 
por la variedad Criollo, que explica una continua acumulación de asimilados en la última etapa de crecimiento.

\section{Agradecimientos}

Los autores agradecen a la Dirección de Investigaciones (DIN) de la UPTC, por el apoyo financiero, en marco del proyecto SGI 1575 (convocatoria DIN 005, proyectos externos regionales, 2014) "Caracterización morfoagronómica y fenológica de lulo (Solanum quitoense Lam.) en la provincia de Neira, departamento de Boyacá".

\section{Referencias}

(1) Heiser C. The relationships of the naranjilla, Solanum quitoense Lam. Biotropica, 1972; 4: 7784. DOI: http://dx.doi.org/10.2307/2989729.

(2) Acosta O., Pérez A., Vaillant F. Chemical characterization, antioxidant properties, and volatile constituents of naranjilla (Solanum quitoense Lam.) cultivated in Costa Rica, 2009; 59(1): 88-94.

(3) Agronet. Sistema de Estadísticas Agropecuarias. Producción de lulo en Colombia 20102013 Recuperado de: http://207.239.251.112/ www/htm3b/ReportesAjax/VerReporte.aspx.

(4) Lobo M., Medina C. Lulo (Solanum quitoense Lam.). In: Caracterizacao de frutas nativas da América Latina. FUNEP. 2000; 41-43. En: Edicao comemorativa do $30^{\circ}$ Aniversario da Sociedade Brasileira de Fruticultura.

(5) Bermeo A. Caracterización de la variabilidad morfológica y algunos atributos químicos de los frutos en una colección de lulo (Solanum quitoense Lam.) y especies relacionadas de la sección Lasiocarpa, Tesis de titulación, Facultad de ciencias agropecuarias, Universidad Nacional de Colombia, Medellín, 2005; 28 p.

(6) Struik PC, Yin X., de Visser P. Complex quality traits: now time to model. Trends in Plant
Science. 2005; 10: 513-516. DOI: http://dx. doi.org/10.1016/j.tplants.2005.09.005.

(7) Génard M., Bertin N., Borel C., Bussières P., Gautier H., Habib R., Léchaudel M., Lecomte A., Lescourret F., Lobit P., Quilot B. Towards a virtual fruit focusing on quality: modelling features and potential uses. Journal of Experimental Botany. 2007; 58: 917-928. DOI: http:// dx.doi.org/10.1093/jxb/erl287.

(8) Liu H-F., Génard M., Guichard S., Bertin N. Model-assisted analysis of tomato fruit growth in relation to carbon and water fluxes. Journal of Experimental Botany. 2007; 58: 3567-3580. DOI: http://dx.doi.org/10.1093/jxb/erm202.

(9) Martre P., Bertin N., Salon C., Génard M. Modelling the size and composition of fruit, grain and seed by process-based simulation models. New Phytologist. 2011; 601618. DOI: http://dx.doi.org/10.1111/j.14698137.2011.03747.x.

(10) Bertin N., Génard M., Fishman S. A model for an early stage of tomato fruit development: cell multiplication and cessation of the cell proliferative activity. Annals of Botany. 2003; 92: 65-72. DOI: http://dx.doi.org/10.1093/ aob/mcg111.

(11) Bramardi S. Modelos de predicción de tamaño de frutos a la cosecha en base a mediciones sucesivas durante el período de crecimiento. Peras cvs. William`s y Packham`s Triumph en la región del Alto Valle de Río Negro y Neuquén. Escuela para graduados Convenio Facultad de Agronomía- UBA e INTA. 2005.

(12) Ostle B. Estadística aplicada. México, D. F.: Limusa. 1986. Montgomery D. Diseño y Análisis de Experimentos. México: Grupo Editorial Iberoamérica. 1991. Calvo H., González A., Pérez B. Manual de Modelos no Lineales en los Ámbitos Agronómico, Ganadero y Forestal. Madrid, España: Ministerio de Agricultura, Pesca y Alimentación. Instituto Nacional de Investigación y Tecnología Agraria y Alimentaria. 1994. 
(13) Montgomery, D. Diseño y Análisis de Experimentos. Ed. Grupo Editorial Iberoamérica. México. 1991. 589 p.

(14) Calvo, H.; González, A.; Pérez, B. Manual de Modelos no Lineales en los Ámbitos Agronómico, Ganadero y Forestal. Ed. Ministerio de Agricultura, Pesca y Alimentación. Instituto Nacional de Investigación y Tecnología Agraria y Alimentaria. Madrid, España. 1994. 110 p.

(15) Casierra F., Barreto V. y Fonseca O. Crecimiento de frutos y ramas de duraznero (Prunus persica L. Batsch, cv. 'Conservero') en los altiplanos colombianos. Agronomía Colombiana, 2004; 22(1): 40-45.

(16) Salisbury F., Ross C. Fisiología vegetal. Editorial Iberoamericana. 1994.

(17) Barceló C., Rodrigo G., Sabater G., Sánchez R. Fisiología vegetal. Madrid: Pirámide, 2001.

(18) Grange R. Crecimiento del fruto. En: Azcón-Bieto J. y M. Talón (eds.): Fisiología y bioquímica vegetal. Madrid: Interamericana-McGraw-Hill. 1996; p. 449-462.

(19) Gardner FP, Pearce RB, Mitchell RL. Physiology of crop plants. Blackwell Publishing Company, lowa. 2003.

(20) Hunt R. Basic growth analysis. Plant growth analysis for beginners. Unwin Hyman, Boston. 1990. DOI: http://dx.doi.org/10.1007/978-94010-9117-6.

(21) Hunt R. Growth analysis, individual plants. En: Thomas B., D. J. Murphy and B. G. Murray (Eds.). Encyclopedia of applied plant sciences. London: Academic Press, 2003; 1618 p.

(22) Casierra F., Cardozo M., Cárdenas J. Análisis del crecimiento en frutos de tomate (Lycopersicon esculentum Mill.) cultivados bajo invernadero. Agronomía Colombiana, 2007; 25(2): 299-305.
(23) Manrique L.A. Plant morphology of cassava during summer and winter. Agron. J. 1990; 82(5): 881-886. DOI: http://dx.doi. org/10.2134/agronj1990.0002196200820005 $0007 x$.

(24) Geraud F., Chirinos D., Marín M. Desarrollo de la planta de tomate, Lycopersicon esculentum Miller, cv. Río Grande en la zona del río Limón del estado Zulia, Venezuela. II. Índice de crecimiento relativo, razón de peso foliar y gamma. Rev. Fac. Agron. 1995; 12: 15-23.

(25) Aristizábal L. M. Fisiología Vegetal. Universidad de Caldas. 2003; 306 p.

(26) Cañizares A., Laverde D., Puesme R. Crecimiento y desarrollo del fruto de guayaba (Psidium guajava L.) en Santa Bárbara, Estado de Monagas, Venezuela. Rev. Cient. UDO Agrícola (Venezuela), 2003; 3(1): 38-38.

(27) Tapia M., López X., Galleti L., Berger H. Caracterización del crecimiento y desarrollo del fruto de melón (Cucumis melo var. Reticulatus Naud.) cv. Topscore. Agri. Téc. (Chile). 1998; 58(2): 93-102.

(28) Avanza M., Bramardi S., Mazza M. Statistical models to describe the fruit growth pattern in sweet orange Valencia late. Span. J. Agric. (Spain). 2008, 6(4):577-585. DOI: http://dx. doi.org/10.5424/sjar/2008064-352.

(29) Coombe B. The development of fleshy fruits. Annu. Rev. Plant Physiology (USA). 1976, 27: 207-228. DOI: http://dx.doi.org/10.1146/annurev.pp.27.060176.001231.

(30) Rojas P., Pérez M., Colinas M., Sahagún J., Avitia E. Modelos matemáticos para estimar el crecimiento de chile manzano. Revista Chapingo, serie Horticultura (México). 2008; 14(3): 289-294.

(31) Casierra F., Cardozo M. Análisis básico del crecimiento en frutos de tomate (Lycopersicon esculentum mill, cv. 'quindío') cultivados 
a campo abierto. Rev. Facultad Nacional de Agronomía, 2009; (62): 4815-4822.

(32) Mazorra M., Quintana A., Miranda D., Fisher G., Chaparro M. Aspectos anatómicos de la formación y crecimiento del fruto de uchuva Physalis peruviana (Solanaceae). Acta Biol. Colomb. 2006; 11(1): 69-81.

(33) Flórez V., Fernández A., Miranda D., Chaves B., Guzmán J. Avances sobre fertirriego en la floricultura colombiana. Bogotá: Unibiblos. 2006.

(34) Rodríguez A., Sosa D. Caracterización de la diversidad genética de lulo (Solanum quitoense L.) en el municipio de Pachavita-Boyacá. Trabajo de grado Ingeniero Agrónomo. Universidad Pedagógica y Tecnológica de Colombia. 2015; 63 p.

(35) Opara L. Fruit growth measurement and analysis. Hort. Rev., 2000; 373-431.

(36) Iglesias D. J., Cercós M. Colmenero-Flores J.M.; Naranjo M.A., Ríos G., Carrera E., Ruiz-Rivero O., Lliso I., Morillon R., Tadeo F.R. y Talón M. Physiology of citrus fruiting. Braz. J. PlantPhysiol. 2007; 19(4): 333-362. DOI: http://dx.doi. org/10.1590/S1677-04202007000400006.

(37) Kays S. Postharvest biology. Athens (Georgia): Exon Press, 2004; 468 p.
(38) Díaz J. y Manzano J. Calidad en lulo (Solanum quitoense Lam.) almacenados a diferentes temperaturas. Interam. Soc. Trop. Hort. 2002; 27-28.

(39) Balaguera H. Estudio del crecimiento y desarrollo del fruto de champa (Campomanesia lineatifolia Ruiz \& Pav.) y determinación del punto óptimo de Cosecha. Tesis de Maestría, Universidad Nacional de Colombia, Bogotá, 2011; 152 p.

(40) Yuri J.A., Gónzalez J., Verdugo J. y Del Pozo A. Responses of fruit growth, quality and productivy to crop load in Apple cv. Ultra red Gala/MM111. Sci. Hortic. 2011; 127: 305312.

(41) Molina-Ochoa M.J., Vélez-Sánchez J.E. y Galindo-Egea A. Resultados preliminares del efecto del riego deficitario durante el período de crecimiento rápido del fruto de pera (var. Triunfo de Viena) en la producción y calidad del fruto. Rev. Col. Cienc. Horti. 2015; 9(1): 38-45.

(42) Álvarez-Herrera J.G., Galvis J.A. y Balaguera López H.E. Determinación de cambios físicos y químicos durante la maduración de frutos de champa (Campomanesia lineatifolia $R$ y P.) Agron. Colomb. 2009; 27(2): 253-259. 